\title{
Target tyranny
}

\author{
Andrew Dicker MB MSc
}

J R Soc Med 2004;97:496-497

General practitioners (GPs) have been galvanized into showing an uncharacteristic interest in the content of the surgery computer software. The only way to benefit from the financial incentives built into the new General Medical Services contract is by optimizing the input of data about people who are included in the Department of Health (DoH) targets. This sudden preoccupation with the need to update the data has nothing to do with the care of the patients and everything to do with maximizing income.

It was Kenneth Clarke who, as Secretary of State for Health, in 1990 replaced the 'fee for service' payment system for primary and secondary prevention with targets, the attaining of which attracted income. During the past 14 years the target culture has invaded the planning of health services, but in primary care the financial incentives applied only to preventive measures in the well populationimmunization programmes and cervical cancer screening. That was until 1 April 2004, with the implementation of the new contract between GPs and primary care trusts (PCTs). The target strategy now applies to selected groups of patients with medical diagnoses.

It is a poor reflection on the profession that no serious ethical objections have been made to the invasion of working practices by the target-hitting culture. It is a paternalistic policy, introduced at a time when the paternalistic tradition, so disastrously cultivated by doctors during the twentieth century, shows genuine signs of being replaced by a more humanistic approach. The target policy is paternalistic because of the assumption that some good will be achieved, for which there is no evidence (but doctors know best), while ignoring the need for the consent of the patients to be partners in the endeavour to pay doctors more. This is not an ethical way to provide healthcare.

Little or nothing is learnt from history. With the cervical screening programmes, women have come under pressure to accept interventions when there was scant evidence that they would be beneficial. There were even stories of doctors threatening to remove patients from their lists if they did not comply. Now, data relating to a host of illness states are included in the new percentage targets which, if met, will mean more money for doctors. The conditions

93 Cambridge Street, London SW1V 4PY, UK

E-mail: a.dicker@imperial.ac.uk and diseases are heart disease, hypertension, diabetes, asthma, epilepsy, schizophrenia, bipolar disorder and hypothyroidism. It would be more ethically acceptable if remuneration was linked to demonstrating that people with these conditions were being actively managed. But that is not the case. Remuneration is linked to computerized data that theoretically demonstrate how effectively the disease is being treated. The profession has signed up to an intensely paternalistic, public-health-driven system. The old tension between population medicine and personal doctoring has never been greater. Already the word on the clinic corridor is that, if the test results are not within the target range, the patient will politely be asked to find another doctor.

The responsibility for this state of affairs is hard to locate. The Department of Health and the GP negotiators devised the convolutions of the new contract. The DoH passed the responsibility of implementing the contract to the primary care trusts, and the negotiators advised the GPs to accept the new terms, with their financial attractions.

The doctors now find themselves undertaking a huge task including measurements and blood tests performed from motives other than the welfare of the patient. Moreover, patients will be under pressure to take more and more pills in the effort to get blood sugar levels, blood pressures, cholesterol levels and so forth low enough to be entered into the consecrated part of the computer database where the right sort of data will qualify for payment. In reality, many practices are training suitable members of staff to be healthcare assistants (HCAs) who will do the work of biometric measurement and data entry, with a doctor somewhere in the background to offer advice if a number falls outside the target range. This has been very good for staff morale since it has extended the role of people who were previously doing reception work or administration.

If some benefit to the health of the nation were likely to accrue from this coercive style of illness management, doctors would need a very good reason not to cooperate. In the absence of objective evidence of benefit, however, GPs are working in a sort of moral vacuum, driven by contractual opportunity and a massive investment of public money in computers to collect and count the data. Moderate paternalism can often be justified by the fundamental desire to do good, but an ethical approach to the contract demands that doctors, nurses and their assistants make clear the mixture of motives: 'your cooperation may 
improve not only your health but also the practice finances'. Alternatively, the waiting room might display performance figures - say, glycated haemoglobin in the diabetic population, with the slogan 'Have you done your bit to make these figures lower? Be sure to take your pills!' Transparency would also be aided by regular declarations of earnings from specific areas of clinical activity.
An ill-thought-out contract, seriously wanting in ethical design, might yet be made a moral instrument by means of which both patients and doctors could benefit. The dissemination of proper information, to make the participation of the patients a more ethical process, might spare doctors future accusations that good medicine has been driven out by Mammon. 